\title{
Analyst
}

CORRECTION

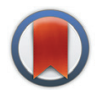

CrossMark

Cite this: Analyst, 2015, 140, 1356

\section{Correction: Imaging mass spectrometry of a mouse brain by tapping-mode scanning probe electrospray ionization}

\author{
Yoichi Otsuka, ${ }^{\star a}$ Junpei Naito, ${ }^{a}$ Shuya Satoh, ${ }^{a}$ Masafumi Kyogaku, ${ }^{a}$ \\ Hiroyuki Hashimoto ${ }^{a}$ and Ryuichi Arakawa ${ }^{b}$
}

DOI: 10.1039/c4an90101k

www.rsc.org/analyst
Correction for 'Imaging mass spectrometry of a mouse brain by tapping-mode scanning probe electrospray ionization' by Yoichi Otsuka et al., Analyst, 2014, 139, 2336-2341.

On pp. 2338 and 2339, there are errors in the captions to Fig. 2 and Table 1, where the ion images and assignments for the major ion peaks shown, respectively, are identified as being obtained from a rat brain section. This is not correct and we would like to confirm that the images and peak data were obtained from a mouse brain section. Correct versions of Fig. 2 and Table 1 are shown here below.

\footnotetext{
${ }^{a}$ Frontier Research Center, Canon Inc., 30-2 Shimomaruko 3-chome, Ohta-ku, Tokyo 146-8501, Japan. E-mail: otsuka.yoichi@canon.co.jp; Tel: +81-3-3758-2111

${ }^{b}$ Department of Chemistry and Materials Engineering, Faculty of Chemistry, Materials, and Bioengineering, Kansai University, 3-3-35 Yamatecho, Suita, Osaka 564-8680, Japan.E-mail: arak@kansai-u.ac.jp; Tel: +81-6-6368-0781
} 

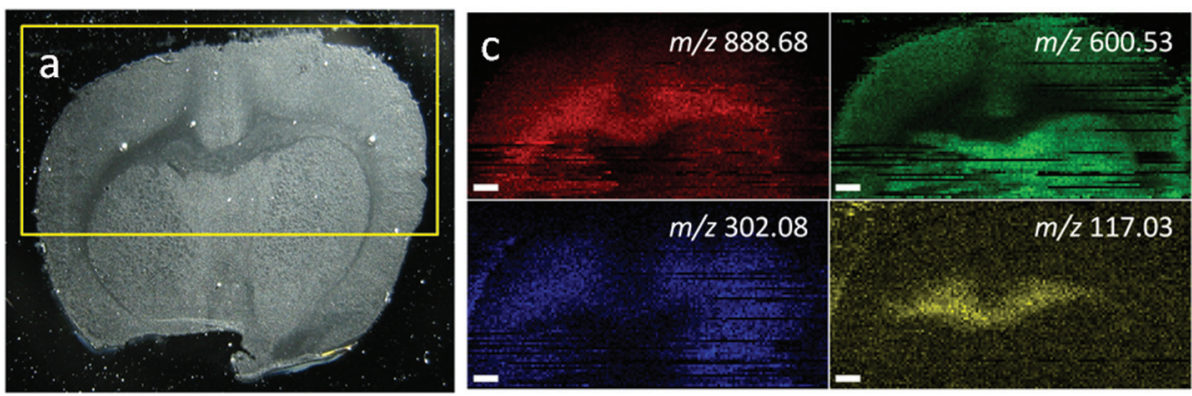

b
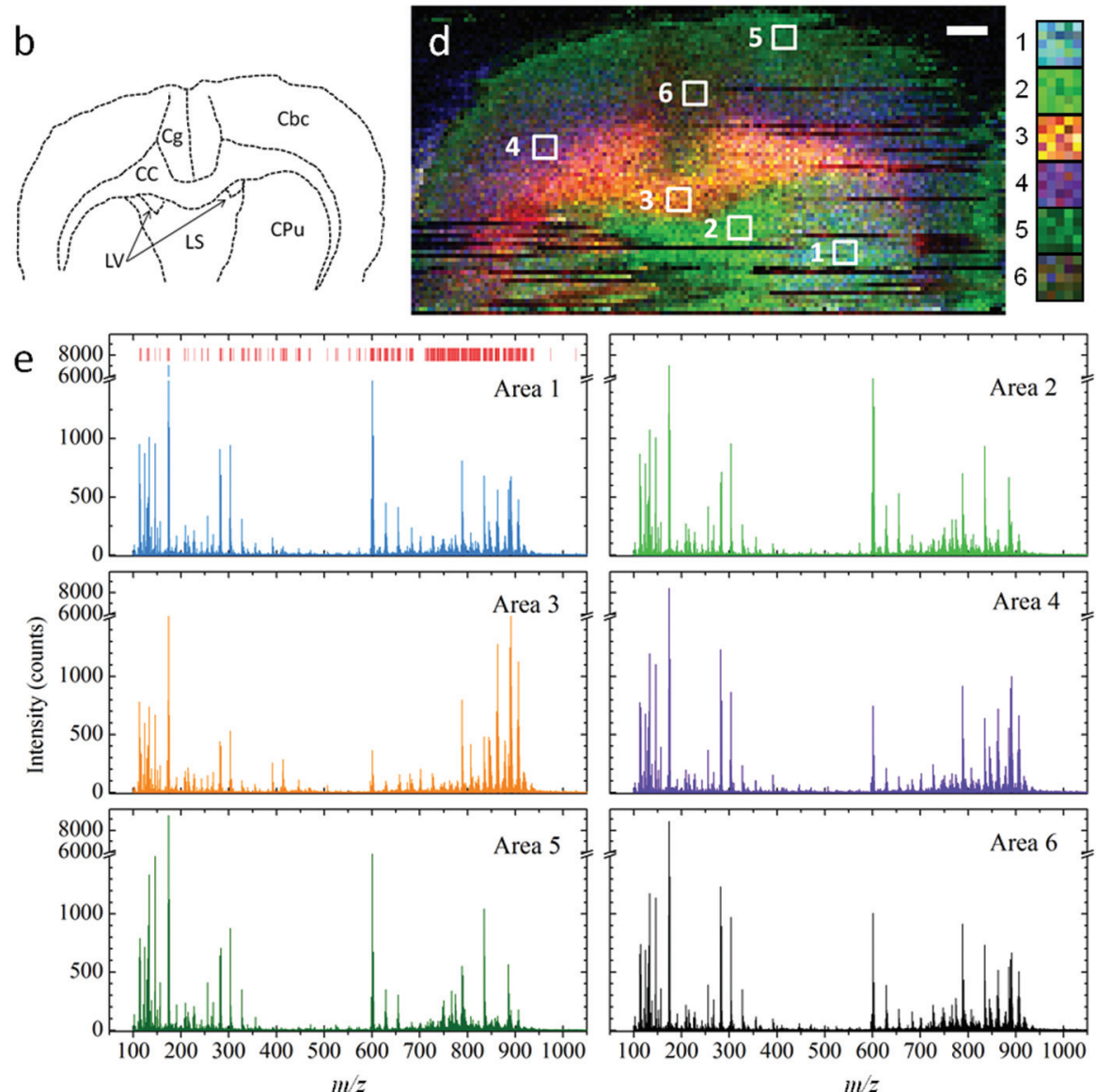

Fig. 2 Negative-ion mode SPESI-MS of the mouse brain section. (a) Optical image of a coronal mouse brain section. (b) Diagram delineates the major brain anatomical regions such as corpus callosum (cc), cerebral cortex (Cbc), caudate putamen (CPu), lateral septum (LS), lateral ventricle (LV) and cingulate cortex (Cg). (c) Selected ion images of specific molecules at the area of t-SPESI measurement. The $\mathrm{m} / \mathrm{z}$ for each image is noted inside. (d) An overlaid image of 4 images in (c). Six regions of interest (ROI) were selected for the comparison of mass spectra. All ROI were magnified as shown on the right side. (e) SPESI mass spectra of six ROI. The ions derived from the brain sections are shown as the red line in the mass spectrum of area 1. Scale bar: $1 \mathrm{~mm}$. 
Table 1 The assignments for the major ion peaks of mouse coronal sections using t-SPESI ${ }^{a}$

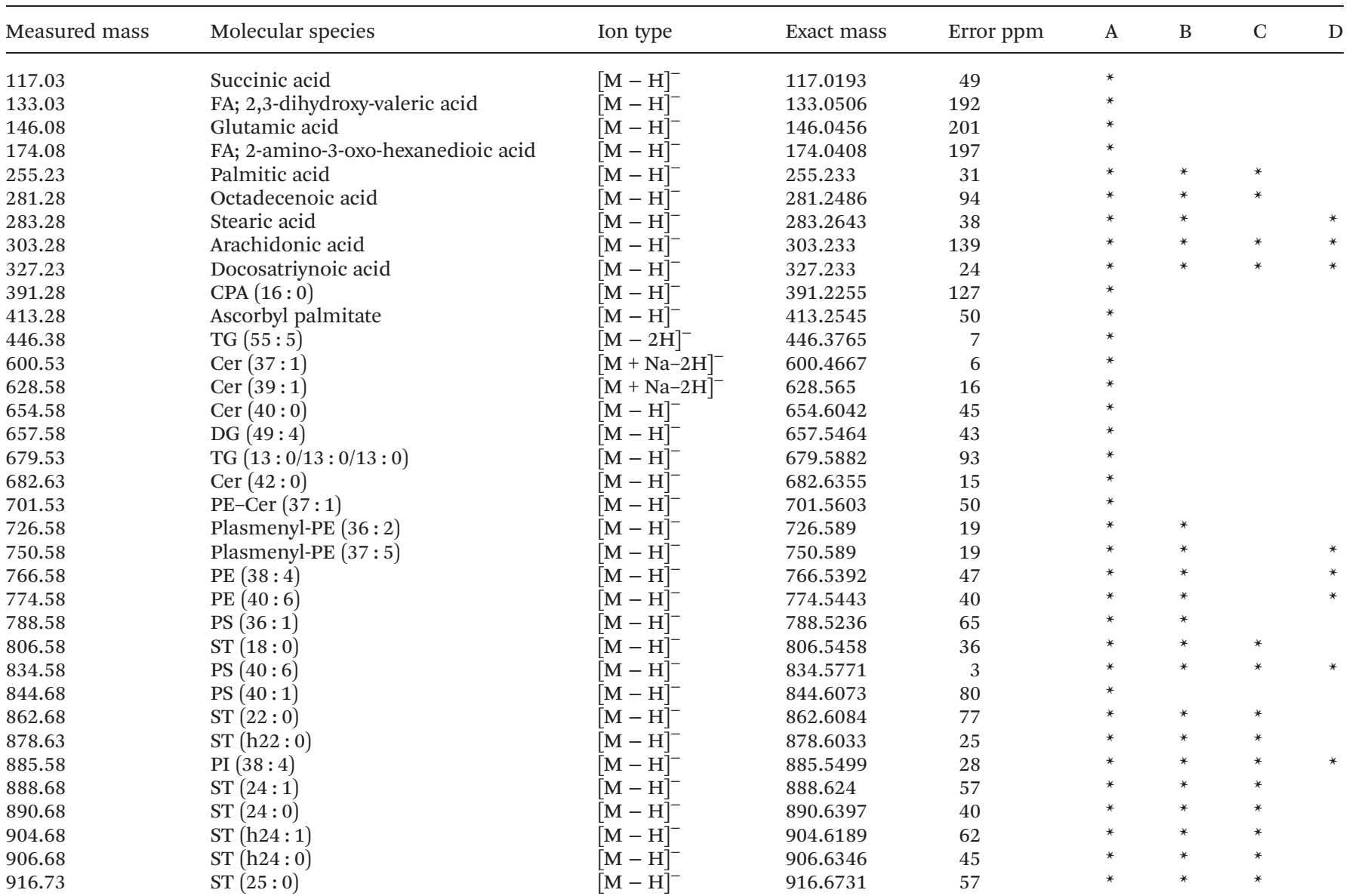

${ }^{a}$ (A) Tentatively assigned form database. (B) Peaks reported by Wiseman et al. (ref. 15). (C) Peaks reported by Eberlin et al. (ref. 14). (D) Peaks reported by Janfelt et al. (ref. 16). Cer, ceramide; DG, diacylglycerols; FA, fatty acid; PE, phosphoethanolamine; PS, phosphoserine; ST, sulfatide; TG, triacylglycerols. 